\title{
Crystal-like high frequency phonons in the amorphous phases of solid water
}

\author{
H. Schober ${ }^{1}$, M.M. Koza ${ }^{2}$, A. Tölle ${ }^{2}$, C. Masciovecchio ${ }^{3}$, F. Sette ${ }^{4}$, and F. Fujara ${ }^{5}$. \\ ${ }^{1}$ Institut Laue-Langevin, F-38042 Grenoble Cedex, France. \\ 2 Fachbereich Physik, Universität Dortmund, D-44221 Dortmund, Germany \\ 3 Sincrotrone Trieste, Area Science Park, I-34017, Trieste, Italy. \\ 4 European Synchrotron Radiation Facility, F-38043 Grenoble Cedex, France. \\ 5 Technische Universität Darmstadt, Hochschulstr 6, D-64289 Darmstadt, Germany.
}

(November 5, 2018)

\begin{abstract}
The high frequency dynamics of low- $(L D A)$ and high-density amorphous-ice $(H D A)$ and of cubic ice $\left(I_{c}\right)$ has been measured by inelastic X-ray Scattering (IXS) in the $1 \div 15 \mathrm{~nm}^{-1}$ momentum transfer $(Q)$ range. Sharp phonon-like excitations are observed, and the longitudinal acoustic branch is identified up to $Q=8 \mathrm{~nm}^{-1}$ in $L D A$ and $I_{c}$ and up to $5 \mathrm{~nm}^{-1}$ in $H D A$. The narrow width of these excitations is in sharp contrast with the broad features observed in all amorphous systems studied so far. The "crystal-like" behavior of amorphous ices, therefore, implies a considerable reduction in the number of decay channels available to sound-like excitations which is assimilated to low local disorder.
\end{abstract}

PACS numbers: 61.12.Ld, 63.50.+x, 64.70.Kb, 78.70.Ck

Amorphous polymorphism, i.e. the existence of two or more amorphous states in the phase diagram of a chemical substance, has recently attracted wide interest from the scientific community [1]. In water, amorphous polymorphism has received particular attention as it was associated with a possible phase separation in the deeply undercooled liquid [2] - this is suggested by the idea that the two amorphous water phases may be identified with the glassy forms of two liquid phases. In systems like water, the ergodic to non-ergodic transition from the undercooled liquid to the glass cannot be studied continuously as a function of temperature due to homogeneous crystallization. The glassy nature of the amorphous ice phases can, therefore, only be established indirectly, and this has contributed to raise controversies on the exact nature of the phase-diagram of water, and on the inter-relations among the different stable and metastable phases of this molecule [3].

The growing perception that the glassy state is a very general state of condensed matter justifies the many experimental, theoretical and simulation studies performed on glasses. Among them, there is a growing interest in the study of the collective dynamical properties at high frequency, i.e. in the wavelength regime approaching the interparticle distances. Here one expects that either a continuum description, valid in the hydrodynamic limit, or the ordered-medium (phonon) picture developed for crystalline materials will fail as a consequence of the disorder. The deviations can then be used to characterize and better understand the glassy state. More specifically, there is a number of dynamic signatures which are associated with the glassy state [4]. At very low temperatures two-level states give rise to tunneling phenomena [5]. At higher temperatures disorder scattering and anharmonicities play an increasingly important role leading to excess densities of states (Boson peak) and relaxational phenomena [6]. Recently, the overdamping of sound waves at high frequency was proposed as a further criteria to characterize the glassy behavior [7]. The study of the collective dynamics of amorphous ice at wavelengths approaching the distance among water molecules may contribute, therefore, to the understanding of the differences between these two phases, and may help to shed light on such issues as their relation to stable crystalline phases or on the existence of two different liquid phases.

In the present letter we present the excitation spectrum of both amorphous forms of water, i.e. high-density $(H D A)$ and low-density amorphous ice $(L D A)$, as determined by high resolution inelastic X-ray scattering (IXS) at momentum transfers $(Q)$ comparable to the inverse of the inter-molecular distance. The experiment has been performed at the very high energy resolution ID16 beamline at the European Synchrotron Radiation Facility in Grenoble. The instrument energy resolution was set to $1.6 \mathrm{meV}$ full width half maximum (FWHM) using $21.748 \mathrm{keV}$ incident photons. The detection system was made up of five independent analyzer systems, displaced among each other by a constant angular offset corresponding to a $Q$-spacing of $3 \mathrm{~nm}^{-1}$. The $Q$ resolution was set to $0.4 \mathrm{~nm}^{-1}$ FWHM. The energy scans at constant $Q$ transfer took about 150', and each $Q$ point was obtained by a typical averaging of 3-5 scans. The transverse dimensions of the beam at the sample were $0.15 \times 0.3 \mathrm{~mm}^{2}$. Further experimental details can be found elsewhere [8].

The $H D A$ sample was obtained by pressurizing hexagonal $\mathrm{D}_{2} \mathrm{O}$ ice $I_{h}$ at $77 \mathrm{~K}$ [9] beyond $10 \mathrm{kbar}$ using the piston-cylinder apparatus described previously [10]. Keeping the sample always at liquid nitrogen temperature, the metastable compound was retrieved from the cell at ambient pressure in mm-size chunks $(\rho=1.17 \pm$ $0.02 \mathrm{~g} / \mathrm{cm}^{3}$ ), and placed into a steel container with two diametrically opposed openings to allow for the passage of the incident and scattered X-ray beam. The effective powder sample thickness along the beam was $\approx 15 \mathrm{~mm}$, 
matching well the X-ray photo-absorption length. Once filled, the container was transferred under a continuous helium flow from the liquid nitrogen bath onto the coldfinger of a precooled closed-cycle refrigerator. Above $\approx 90 \mathrm{~K} \mathrm{HDA}$ transforms with a strongly temperature dependent rate to $L D A\left(\rho=0.94 \pm 0.02 \mathrm{~g} / \mathrm{cm}^{3}\right)$, which, in turns, converts itself into cubic ice $I_{\mathrm{c}}$ at $\approx 140 \mathrm{~K}$. $H D A$ and $L D A$, after proper annealing, were both measured at $60 \mathrm{~K}$, while $I_{c}$ after annealing was measured at $80 \mathrm{~K}$. The similarity of the temperatures allows a direct comparison of the scattering intensities.

The purity of each phase was checked by measuring the respective static structure factors, $S(Q)$, reported in Fig. 1 with a momentum resolution of $0.04 \mathrm{~nm}^{-1}$. Both $H D A$ and $L D A$ samples show the known static structure factor 11] with no signs of Bragg-peaks and, therefore, they are free of crystalline ice XII [12]. There is a pronounced small-angle signal in $H D A$ which disappears upon annealing to $L D A$. Within our energy resolution, its origin is purely elastic as it will be seen by the inelastic scans reported in the following. As only moderate changes in the elastic small angle signal were observed with neutrons 13, it is questionable whether the strong effect observed here is a real bulk property of the $H D A$ sample or whether its origin stems from surface scattering in the powder sample.

A selection of inelastic spectra obtained in the range $Q<8 \mathrm{~nm}^{-1}$ are reported in Fig. 2 and Fig. 3. In both amorphous ice phases and in the cubic crystalline phase there are well pronounced resonances. Increasing $Q$ one initially observes a single feature with a marked dispersion. At $Q$-values higher than $5 \mathrm{~nm}^{-1}$, the spectra become more complicated by the appearance of additional inelastic features. As X-rays, like neutrons, couple directly to the longitudinal component of the density fluctuations, the dispersive excitation observed from the lowest $Q$-values can be readily identified with the longitudinal acoustic-like branches. In $H D A$ these excitations can be clearly observed up to about $5 \mathrm{~nm}^{-1}$, while, in $L D A$ and $I_{c}$, they can be safely distinguished from the other feature(s) up to $10 \mathrm{~nm}^{-1}$.

In order to derive the energy position, $\hbar \Omega(Q)$, and the width, $\hbar \Gamma(Q)$, of the excitations, they have been fitted by a Lorenzian function convoluted with the experimentally determined instrumental resolution function. This resolution function corresponds to the central peak at $E=0$ in Fig. 2, that, as discussed previously, is due to the static disorder in the samples and to small angle scattering from the sample environment (see Fig. 2 and Ref. [8]). The best fit to the inelastic X-ray scattering data shows that the excitation energy $\hbar \Omega(Q)$ scales linearly with the momentum transfer $Q$ in the limit of small momentum transfers. ¿From the derived linear relation, $\Omega(Q)=c Q$, it is possible to obtain the longitudinal sound velocities $c_{H D A}=3550 \pm 50 \mathrm{~m} / \mathrm{s}, c_{L D A}=3550 \pm 50 \mathrm{~m} / \mathrm{s}$ and $c_{I_{c}}=3750 \pm 50 \mathrm{~m} / \mathrm{s}$, respectively. The differences among these values are small, and this is in agreement with the similar Debye level found in these three materials by neutron scattering experiments [13]. The determination from the best fits of the resonance widths, $\hbar \Gamma(Q)$, is more involved, and is complicated by two facts: $i)$ the absolute values for $\Gamma(Q)$ are highly correlated with the background, and ii), particularly in $H D A$, the features appearing with increasing $Q$ on the low-frequency side of the acoustic-like peaks interfere with the fit. Therefore, no systematic dependence of $\Gamma(Q)$ on $Q$ has been established. In both $L D A$ and $H D A$ one observes, however, that the resonances become broader with increasing $Q$ transfer. In any case care has to be taken in interpreting such broadening because the line shape of the resonances is unknown. For a single phonon it could be assumed Lorenzian reflecting the finite lifetime of the excitation. However, when taking a powder average the line shape acquires a non-analytic form even for the crystal due to the anisotropy within the multidimensional dispersionsheet.

Despite the difficulty to give full account of the observed broadening in the measured spectra, one can deduce from Figs. 2 and 3 the following important observation: As seen by the naked eye, the width of the resonances remains by far smaller than the excitation energy, $\hbar \Omega(Q)$, for all the considered $Q$-values. This result is very different from the observation made so far in other glassy systems. There, in fact, one observes an acoustic phononlike resonance with a linear dispersion of $\Omega(Q)$ vs $Q$ and a quadratic dispersion of $\Gamma(Q)$ vs $Q$ up to a value $Q=Q_{m}$. $Q_{m}$ is defined by the relation $\Omega\left(Q_{m}\right) \approx \Gamma\left(Q_{m}\right)$. At $Q$ larger than $Q_{m}$ it is no longer possible to observe well defined excitations and the inelastic part of the spectrum is, at the most, a broad and structureless background.

Let us take the position, $Q_{M}$, of the first sharp diffraction peak as an indicator for the extent of structural correlations in the two amorphous phases. This allows us to define a pseudo Brillouin zone 14] which extends to $Q_{M} / 2 \approx 10 \mathrm{~nm}^{-1}$ in $H D A$, and to $Q_{M} / 2 \approx 8 \mathrm{~nm}^{-1}$ in $L D A$. The quantity $Q_{m} / Q_{M}$ has so far always been found smaller than 0.5 [7]. In the two amorphous ice phases studied here, excitations are very well defined up to $Q$-transfer values approaching $Q_{M}$, and, at least in $L D A$, the longitudinal acoustic-like branch can be identified very well at least up to $Q=8 \mathrm{~nm}^{-1}$. In Ref. [7], it has been suggested the possible existence of a relation between the value of $Q_{m} / Q_{M}$ and the degree of fragility of the considered glass [15]. The high value of $Q_{m} / Q_{M}$, which seems to approach unity in these two glasses, would imply that amorphous ices, and especially $L D A$, are the extreme end of fragile glasses. This is in contradiction to the view that water passes through an inflection in the deeply supercooled region where the liquid behavior changes from extremely fragile to strong [16, 17].

A further observation coming from the analysis of the spectra in Fig. 2 and 3 is that the spectra of $L D A$, at all 
the considered $Q$-values, are very similar to those measured in ice $I_{c}[18]$. In $H D A$ the resemblance is less pronounced [19]. The similarity of $L D A$ and $I_{c}$ holds not only for the resonances assigned to the acoustic mode, but also for the excitations at $\approx 7 \mathrm{meV}$, which appear at higher $Q$ and recall the transverse dynamics found in ice $I_{h}$ and liquid water [8]. In fact, as in these systems, the excitations set in around $5 \mathrm{~nm}^{-1}$ and, as seen in Fig. 3, they become more pronounced beyond $Q_{M}$. The translational part of the density-of-states for $I_{h}, I_{c}$ and $L D A$, as obtained from INS spectra [13], is peaked around 6.5 $\mathrm{meV}\left(\mathrm{D}_{2} \mathrm{O}\right)$. It is, therefore to be expected, that the INX spectra show excitations in this energy region at low $Q$ due to Umklapp processes. These processes take place via the Bragg peaks in the crystalline state and via the static structure factor in the case of $L D A$ [20].

Despite the high definition of the low $Q$ excitations in the two amorphous ice phases when compared to other glasses, one recovers a clear indication of the disordered character from the evolution of the inelastic spectrum at larger $Q$ values. The spectra of $L D A$ and $H D A$ are less structured, lacking the sharp features observed in ice $I_{c}$.

To conclude, we reported on an IXS measurement of the $S(Q, E)$ of the two known phases of amorphous ice. This has allowed to show that these two states of the water molecule possess a surprisingly crystal-like dynamic response. In both $H D A$ and $L D A$ the sound wave excitations are well defined. These experimental findings are in sharp contrast to the results found so far in other glasses, glass forming materials, liquids, dense gases and disordered materials in general. In these systems, in fact, an important broadening has always been observed in the inelastic part of the dynamic structure factor $S(Q, E)$. In this $Q$-region the scattering experiment becomes sensitive to the topological disorder which opens decay channels for sound excitations in addition to those available in the crystal. These channels are found practically absent in the case of the amorphous ice phases indicating a very low degree of local disorder. Structural results are controversially discussed and to date do not give a clear picture of the topology [21,22]. Experimentally only the pair correlation functions are directly accessible. Higher order correlations, among these the orientational correlation function, must be obtained in an indirect way [23] e.g. via the dynamic response. We deduce from our data highly intact hydrogen bond networks both in $L D A$ and to some lesser degree equally in $H D A$. Although an intact network in itself is no warranty for the absence of decay channels - e.g. an infinite random framework of corner-linked $\mathrm{SiO}_{2}$-tetrahedra can undergo large phononassisted distortions [24] - it seems a necessary condition. In $L D A$ this network is perfectly annealed as it is not obtained via a fast quench from the liquid. Due to the constraints of the network the number of states the system can sample on the ps time scale should be small, i.e. there is a small configurational entropy, a view which is compatible with thermodynamic data [17]. $H D A$ is expected to possess a larger configurational entropy, and on this basis one can justify that $H D A$ has more "glassy behavior" than $L D A$.

Apart from structure and bonding the dynamical properties of the water molecule influence the decay of soundlike excitations both in crystalline and amorphous ice. We just want to point to the clear separation of translational and librational bands which independent of the structural details arises from the very small moment of inertia of the water molecule. This separation closes decay channels - e.g. present in $\mathrm{SiO}_{2}$ 25] - involving resonances of acoustic-like and librational modes, and may equally explain the absence of strong excess intensities in the inelastic neutron scattering data [13]. Temperature and the concomitant anharmonicities equally have to be given proper consideration in the discussion [25]. In the end only detailed molecular dynamics calculations on well characterized ensembles combined with experiments on similar systems will be able to unambiguously give the reasons for the crystal like dynamic response of amorphous ice phases.

We acknowledge A. Mermet for his help during the IXS measurements, B. Gorges and R. Verbeni for technical support, F. Sciortino, C.A. Angell and G. Ruocco for useful discussions. We also acknowledge the financial support of the Bundesministerium für Bildung und Forschung under project number 03FU4DOR5.

[1] P.H. Poole T. Grande, C.A. Angell, and P.F. McMillan, Science 275, 322 (1997).

[2] S. Harrington, R. Zhang, P.H. Poole, F. Sciortino, and H.E. Stanley, Phys. Rev. Lett. 78, 2409 (1997).

[3] J.S. Tse, D.D. Klug, C.A. Tulk, I. Swainson, E.C. Svensson, C.-K. Loong, V. Shpakov, V.R. Belosludov, R.V. Belosludov, Y. Kawazoe, Nature 400, 647 (1999).

[4] H.E. Fischer, F.J. Bermejo, G.J. Cuello, M.T. FernándezDiaz, J. Dawidowski, M.A. González, H. Schober, and M. Jimenez-Ruiz, Phys. Rev. Lett. 82, 1193 (1999).

[5] P.W. Anderson, B.I. Halperin, and C.M. Varma, Philos. Mag. 25, 1 (1972).

[6] U. Balucani and M. Zoppi, Dynamics of the Liquid State, (Clarendon Press, Oxford, 1994).

[7] F. Sette, M.H. Krisch, C. Masciovecchio, G. Ruocco, and G. Monaco, Science 280, 1550 (1998).

[8] G. Ruocco and F. Sette, J. Phys: Cond. Matt. 11, R259 (1999), and references therein.

[9] O. Mishima, L.D. Calvert and E. Whalley, Nature, 310, 393 (1984).

[10] M. Koza, H. Schober, A. Tölle, Th. Hanse, and F. Fujara, submitted

[11] M.-C. Bellissent-Funel, J. Teixeira, and L. Bosio, J. Chem. Phys. 87, 2231 (1987). 
[12] M. Koza, H. Schober, A. Tölle, F. Fujara, and T. Hansen, Nature 397, 660 (1999).

[13] H. Schober, M. Koza. A. Tölle, F. Fujara, C.A. Angell, and R. Böhmer, Physica B 241-243, 897 (1998).

[14] D. Caprion, P. Jund, and R. Jullien, Phys. Rev. Lett. 77, 675 (1996).

[15] C.A. Angell, in "Relaxation in Complex Systems" edited by K. L. Ngai and G.B. Wright, NRL p. 3, (Washington, 1984).

[16] K. Ito, C.T. Moynihan, and C.A. Angell, Nature 398, 492 (1999).

[17] F.W. Starr, C.A. Angell, R.J. Speedy, and H.E. Stanley, arXiv cond-mat/9903451.

[18] Cubic ice is not a perfect crystal due to the presence of staking faults (W.F. Kuhs, D.V. Bliss and J.L. Finney, J. Physique 48, C 1631 (1987)). This is an interesting subject in its own, however, without incidence on the discussion of the amorphous phases presented here.

[19] It should be pointed out that while ice $\mathrm{I}_{c}$ is a good reference system for $L D A$ this may not be the case for $H D A$. $H D A$ has supposedly a bonding topology closer related to high density ice forms like ice XII, which is formed under similar conditions 12.

[20] U. Buchenau, Zeitsch. Phys. B 58, 181 (1985).

[21] M.-C. Bellissent-Funel, Europhys. Lett. 42, 161 (1998).

[22] L. Pusztai, Phys. Rev. B 61, 28 (2000).

[23] A.K. Soper, J. Chem. Phys. 101, 6888 (1994).

[24] K. Trachenko, M.T. Dove, K.D. Hammonds, M.J. Harris, and V. Heine, Phys. Rev. Lett. 81, 3431 (1998).

[25] S.N. Taraskin and S.R. Elliott, Phys. Rev. B 59, 8572 (1999).

\section{FIGURE CAPTIONS}

FIG. 1 - Static structure factor of high density $(H D A)$ and low density $(L D A)$ amorphous ice as measured on the inelastic $\mathrm{X}$-ray beamline prior to the inelastic experiments. No signs of Bragg peaks are observed, indicating that both amorphous phases are free of crystalline ice XII contaminations. Note the strong small-angle signal in $H D A$. The inset shows the diffraction pattern of cubic ice.

FIG. 2 - Inelastic X-ray spectra of high density amorphous $(H D A)$, low density amorphous $(L D A)$ and crystalline cubic ice $\left(I_{c}\right)$ at the indicated $Q$ values lying mainly in the first pseudo Brillouin zone. The dashed line is a fit to the sinal using Lorenzian lineshapes convoluted with the resolution function (equally indicated). The solid line represents the inelastic contribution to the total fits. The numbers in brackets on the left of the elastic line give the elastic intensities in arbitrary units. Note the close resemblance of the sharp inelastic response of both amorphous phases to the crystalline phase.

FIG. 3 - Inelastic X-ray spectra of high density amorphous $(H D A)$, low density amorphous $(L D A)$ and crystalline cubic ice $\left(I_{C}\right)$ at high $Q$ values in the second pseudo Brillouin zones. The dashed line gives the resolution function. The elastic intensities are given in brackets. For these high $Q$ values the excitations become part of a broad intensity distribution reminiscent of the density-of-states. 


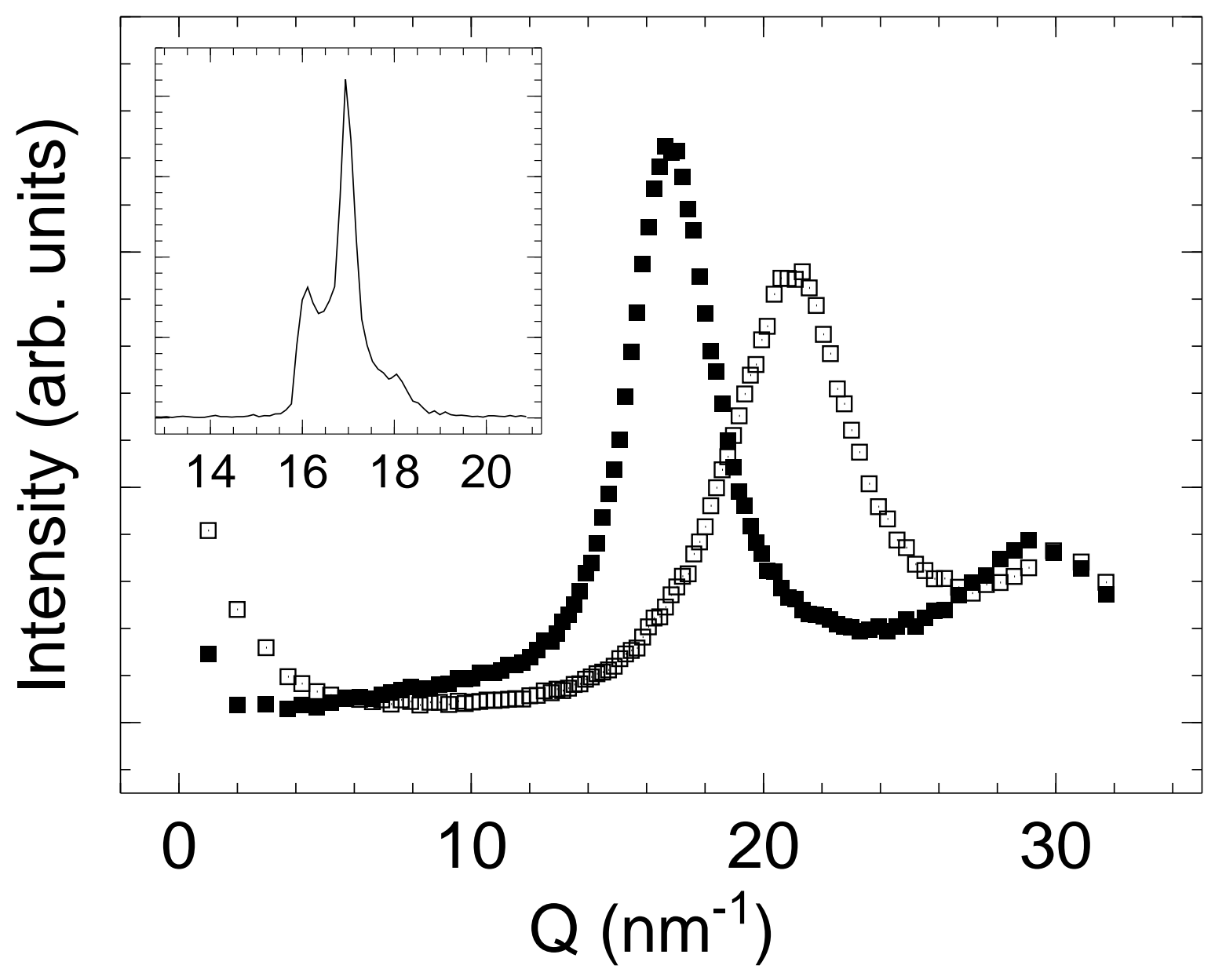

Figure 1 


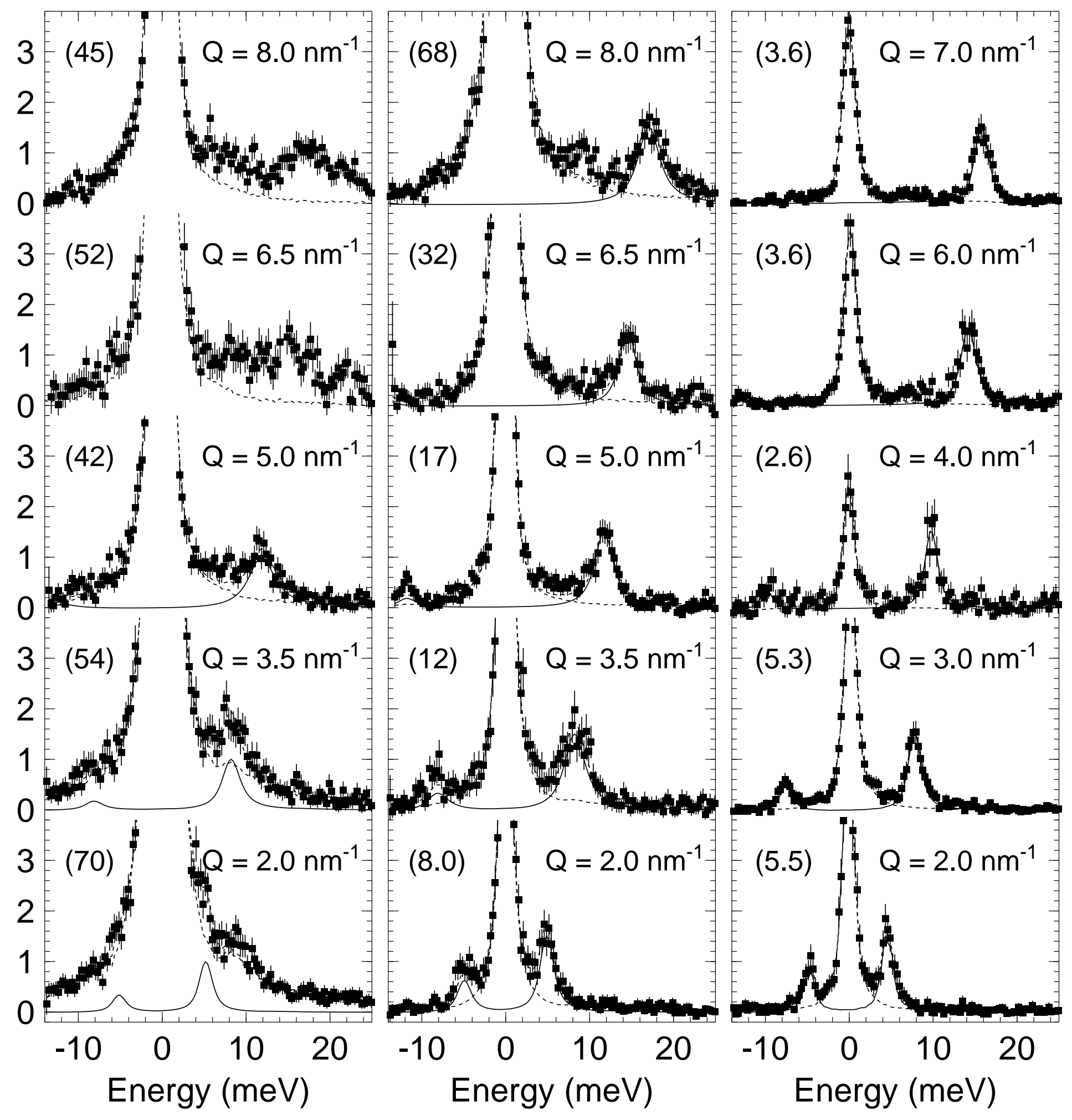

Figure 2 
HDA

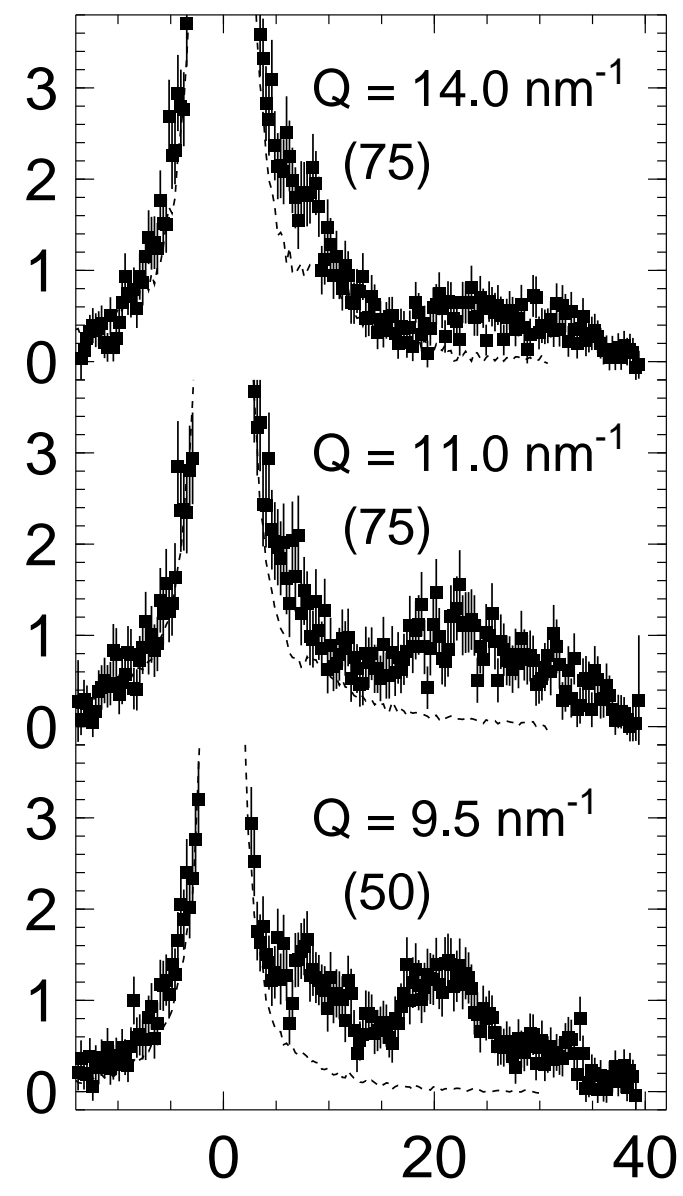

Energy (meV)
LDA

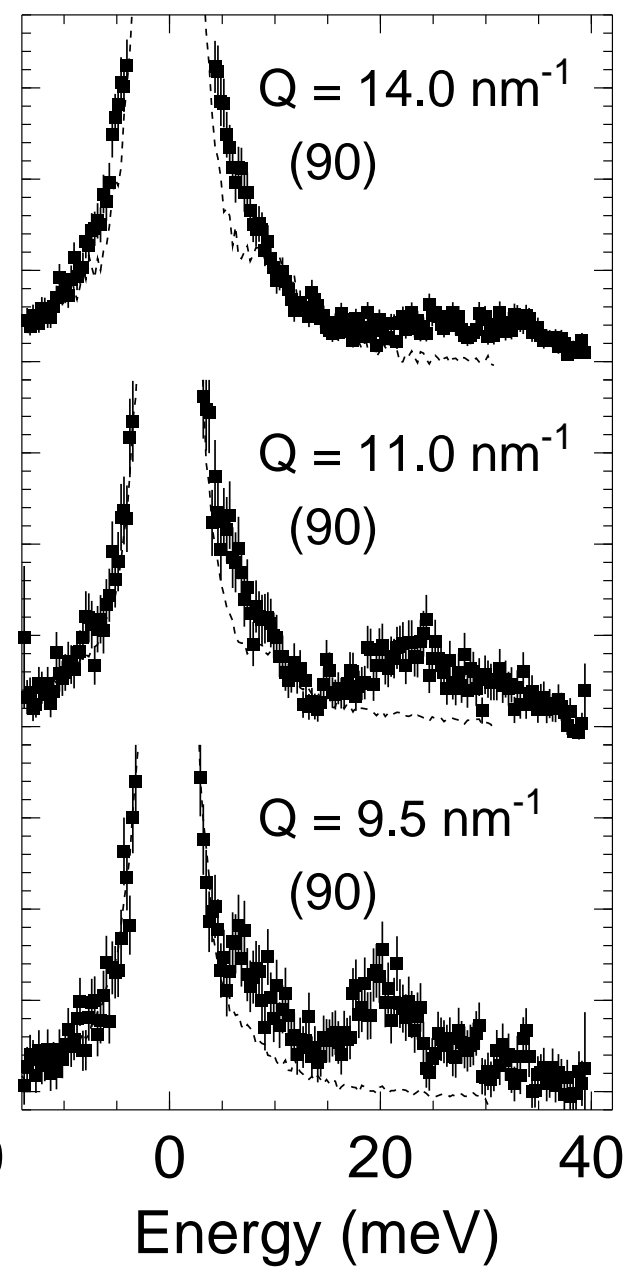

CUB

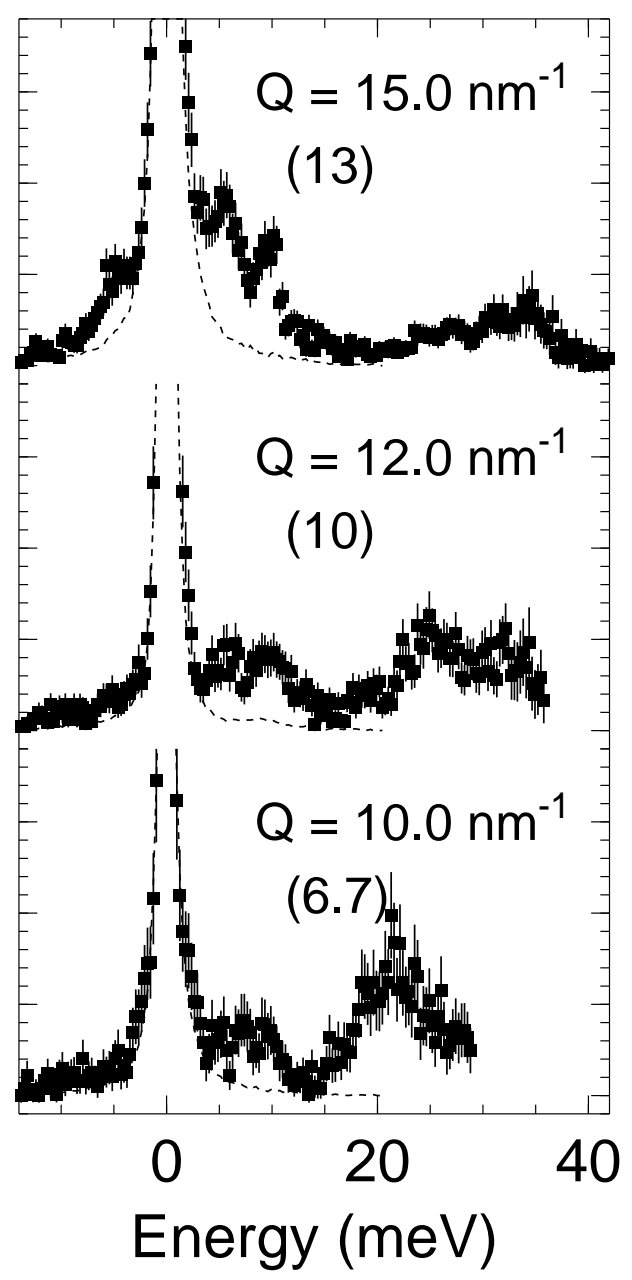

Figure 3 